\title{
Design of Control Scheme for Satellite Ground Application System based on UNIX
}

\author{
Shen Jian, Yang Minying, Shi Yanyang, Li Mingzhe
}

Xi'an Satellite Control Center, China

Keywords: ground application system; architecture; hierarchical scheduling; operation model

\begin{abstract}
To control the satellite in ground, the control scheme of satellite ground application system is designed in this paper. It is responsible for task scheduling, system monitor and system coordinate. As the nerve center of the ground system, the control scheme uses a mixture of C/S and $\mathrm{B} / \mathrm{S}$ architecture based on its characteristics. There are many tasks need be scheduled by the ground application system, such as the time, the scheduled and the data reprocessing. Due to the complexity of data processing process, the operation control subsystem uses a hierarchical scheduling module to reduce scheduling complexity. In addition, the system uses the automatic operation mode, including the development of pre-business operation plan, the distributed task scheduling and the centralized status monitor.
\end{abstract}

\section{Introduction}

The satellite ground application system is a real-time application system. The main task of the system is to be responsible for the control of oceanographic satellite, including the operational management, the production of marine satellite remote sensing and the application service of these produces ${ }^{[1]}$. The ground application system consists of seven subsystems, including operational control communication subsystem, receive and preprocess subsystem, precision classified track subsystem, data processing subsystem, product filing and distribution subsystems, benchmarking and truth inspection subsystems and business operations subsystem ${ }^{[2]}$.

The operational control communication system is composed of three subsystems. And the operational control subsystem (OCS) is the most important subsystem. The primary function of the OCS is to control the entire application system during the business is running. Such as task scheduling and control, monitor of the operation and equipment status, coordinate the business operation between the systems, and complete the control mission ${ }^{[3]}$.

\section{Functional}

The OCS is responsible for the operation control, task scheduling and management. It provides the centralized man-machine monitor UI. The UI can monitor and manage the business process, system equipment and its state. The functional entities diagram of the OCS is shown in figure 1.

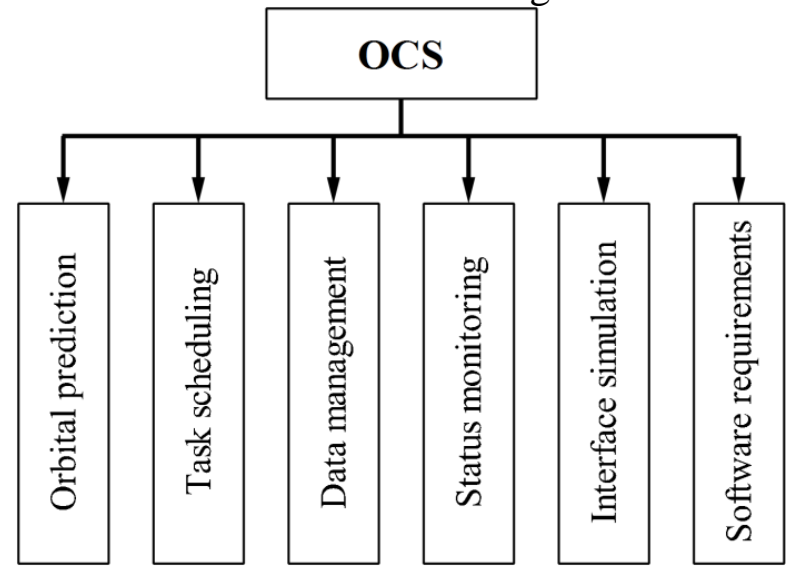

Fig. 1 OCS functional entities 
The main functional of OCS are as follows.

1) Orbital prediction. Such as orbital number acquisition, orbital time calculation, satellite altitude calculation, satellite two-line report calculation, and orbital prediction for other stations.

2) Task scheduling. The scheduling plan for ground application system is generated based on the work plan, the receive plan and the operation scheduling rules. The task scheduling can realize many functions, including satellite remote sensing data transmission, preprocessing, precise orbit determination and processing.

3) Data management. To implement the real-time data transmission between different systems, to establish the operation status database and the configuration parameter, to monitor system resource, to clean up disk periodically, to provide operation status, configuration and management by Web.

4) Status monitoring. The status monitoring is realized hierarchically. The monitoring includes the equipment, the operation status, the ground station, and the remote sensing.

5) Interface simulation. The function simulation including ground stations, communications, preprocessing, processing, archiving and the remote sensing data process.

6) Software requirements. The UI design should based on a unified design style, and a unique system management.

\section{Scheme design}

\subsection{Architecture design}

The OCS uses a mixed style system structure. It is including three-layer C/S architecture and B/S architecture $^{[1]}$. The basic feature of the three-layer architecture is to process the UI, the application and the data respectively. They were implemented by different resource code. The advantage of the architecture is all kinds of modules are independent of each other, with single function and good expansibility ${ }^{[4]}$.

Both C/S and B/S architectures are incorporated into OCS. The combination makes full use of their advantages. The OCS is divided into two parts, including customer software and server software. The server software is divided into business layer software and data layer software according to different functions. The real-time information exchange between server software is implemented on UNIX through using message queues. The customer software is divided into real-time software and data management software based on different data sources. The real-time customer software uses C/S architecture to monitor states. The data management customer software uses B/S architecture to retrieve reality data in a database. The OCS architecture is shown in figure 2.

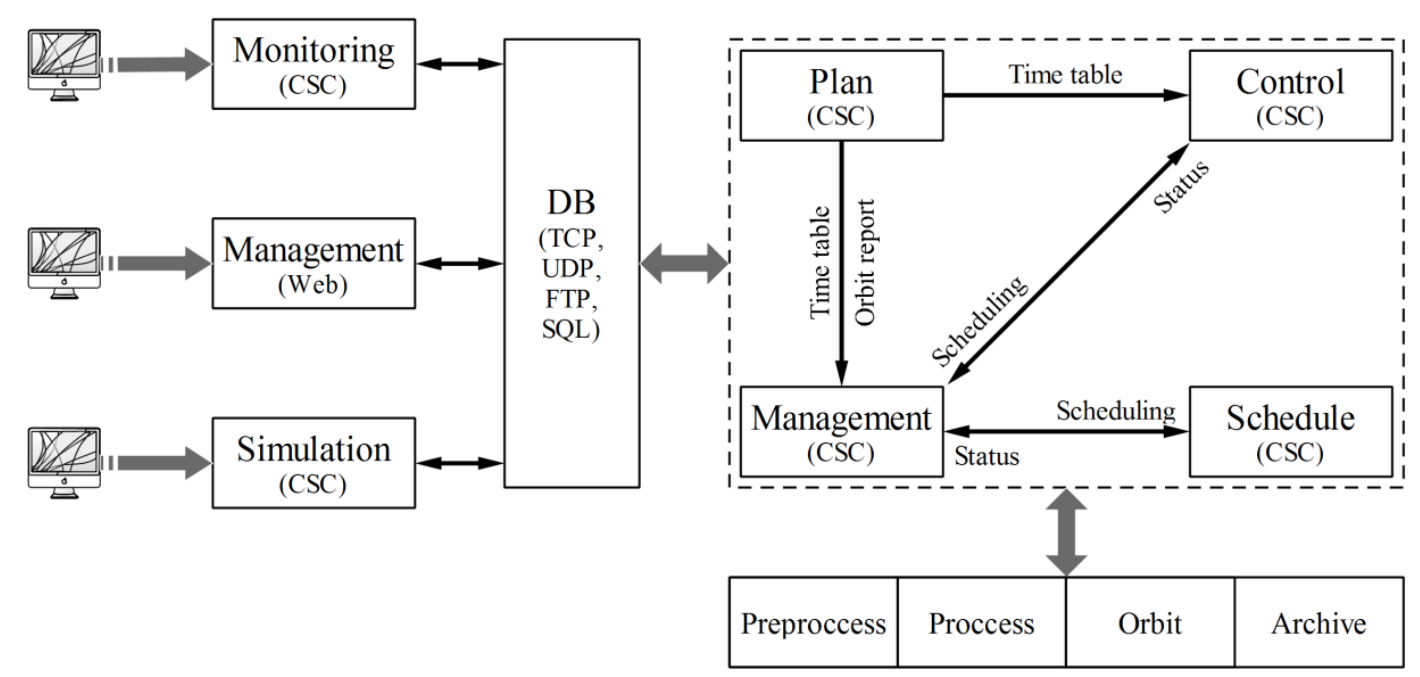

Fig. 2 OCS high level design architecture

\subsection{Operational process}

The process of OCS includes scheduling process and monitoring process. The scheduling 
process is describes the working plan, receiving plan, scheduling plan and generating reports. The monitoring process is mainly used for monitoring the equipments state. These equipments include satellite, ground station, communication subsystem, data processing center and network system etc. These states are collected through the operation control scheduling agent and network management software.

The flow of the operational control communication subsystem is as follows:

1) Receive the telemetry data from satellite, and process the data parallel.

2) Receive the orbital number, and process into standard orbital reports.

3) Forecast the orbit, and make the work plan.

4) Generate the remote control instructions, and send them to satellite.

5) Generate the scheduling plan, send scheduling instructions to subsystem, and receive scheduling status from subsystem.

6) Real-time monitoring of equipment status messages.

7) The information is classified after the operation is completed, analyze statistics and release the information through the Web.

\subsection{Interface design}

OCS is the operation and scheduling center of the maritime satellite ground application system. Therefore, there are software and hardware interfaces between OCS and other subsystem. These interfaces include GS (ground station), DPP (preprocessing subsystem), DCS (communication subsystem), TCS (measurement and control subsystem), PODS (precision orbit subsystem), DPS (processing subsystem), PADS (archive and distribute subsystem), CVS (calibration subsystem) and the human-computer interaction of OCS.

The DPP, DCS, TCS, PODS, DPS, PADS, CVS and internal interface of OCS are transmitting data through network. The data exchange between OCS and TCS through database. The data transmit between OCS and GS by fiber network. The purpose of all these interfaces is to realize the computer networking, visualization and automation.

OCS software interface requirements analysis and design will use interfaces standardized techniques. Declare the software interface through tabulating, hierarchical and progressive. Implementation software interface standardization design and computer aided standardization software interface control. Interface identifiers are used to identify different interfaces, each identifier all correspond to a unique interface.

\subsection{Operation scheduling strategy}

The scheduling policy is critical to a running control system. It will affect process scheduling and fault handling directly. OCS scheduling tasks include real-time scheduling, time scheduling, and data processing scheduling. Due to scheduling objects for OCS are not single and hierarchical, this paper proposed a hierarchical scheduling model ${ }^{[5]}$. Master scheduling is responsible for the scheduling of the main process of the system, and the scheduling agent is responsible for the product generate internal processes for scheduling.

1) Real-time scheduling. Automatic scheduling is implemented in the form of schedule driver and state message driver. Firstly, OCS generates reception according to the orbital prediction results. Then distributes the schedule to the receiving subsystem and the communication subsystem, arrange the receiving plan and data transmission plan for each ground station. At the same time, generate scheduling schedule according to configuration parameters. OCS starts process tracking according to scheduling schedule process. Operation control subsystem scheduling ground application system through multi-level scheduling method, including real-time scheduling process, real-time process tracking and sub-system scheduling agent.

2) Scheduled. The timing scheduling task main include day, week, month, season, year time schedule task. These missions will generate scheduler in advance according to the configuration parameters. Start scheduling plan to track work process according to time constraint. In order not to affect real-time scheduling, the time scheduler is independent of the real-time scheduler.

3) Timeout strategy. When a task is in its scheduled timeout mode because it does not receive the 
status information from the subsystem, it is considered that the task execution is fail. The error handling include check the archival status of the product and ensure all generated products file.

\section{Implementation of software}

\subsection{Software function}

According to the distributed architecture, the OCS software can be divided into OCS_S and OCS_C two software configuration entries. Each of entries can be divided into several program-level components according to the principle of functional cohesion. The CSC functional entities of OCS server software are shown in figure 3.

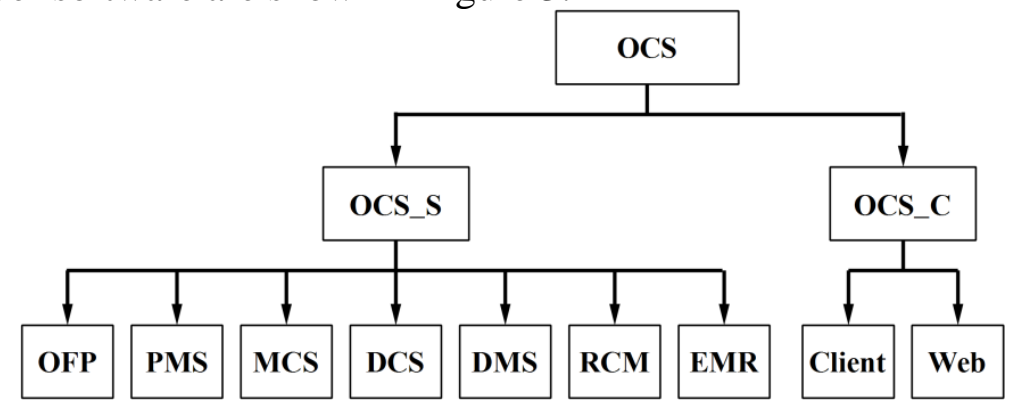

Fig. 3 Functional entities of software

1) Project. Real-time operation control application software.

2) Software configuration (CSCI). Server software component the distributive component (OCS_S) and distributed configuration of soft parts for guest machines component (OCS_C).

3) Procedure (CSC). Orbital forecasting process (OFP), operational planning and dispatch procedures of ground application system (OPS), Operation monitoring information collection and service procedures (MCS), Data communications program (DCS), Data management procedures (DMS), Server run configuration management procedures (RCM), CS Client program (Client), And web management department system program (Web).

\subsection{Software deployment}

Due to OCS is the nerve center of the ground system, the requirements of operating environment is high and reliable. Thus, the OCS server-side program deploys a pair on two hot standby servers. Any one server shut down work, the other can take over automatically. The client is deployed at the monitor as a site. The application deployment details are shown in figure 4.

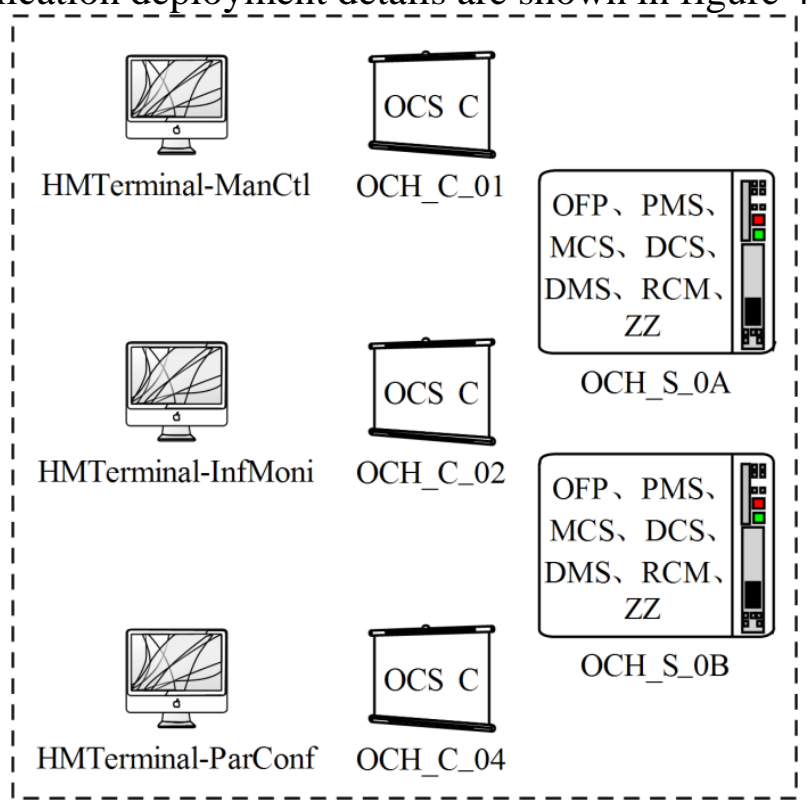

Fig. 4 Software deployment of OCS 
1) Hardware configuration (HWCI). OCS vehicle A (OCH_S_OA), OCS server B (OCH_S_0B), HMTerminal_ManCtl (OCH_C_01), OCS master console, HMTerminal_InfMoni (OCH_C_02), HMTerminal_ParConf (OCH_C_04), OCS parameter the terminal.

2) Computer software configuration (CSCI): OCS server software configuration (OCS_S) And OCS client software configuration (OCS_C).

3) Procedure (CSC): Track forecasting process (OFP), business operation plan and scheduler (PMS), business operations supervisor control information collection and service procedures (MCS), data access program (DCS), data management procedures (DMS), and server running configuration pipe program (RCM).

4) Public operation package: OCS public service operation package (ZZ).

\subsection{Operation mode}

The real-time business of OCS runs in automatic mode. The automatic scheduling strategy includes pre-make operation plan, a distributed planned operation scheduling, and centralized operational status monitoring. The automatic monitoring of the real-time service operation of the satellite ground application system uses the following principle:

The OCS is both send plans and carry out remote monitoring to the ground station and other subsystems. Before performing the data receiving/storage task, the operational plan and orbit tracking documents are sent to the data receiving station by OCS. As the basis of satellite tracking and data reception/transmission, during the implementation of real-time data reception/storage, the data collection stations and the subsystems receives scheduling commands momentarily from OCS, perform the corresponding tasks follow the instructions, and report the performance to OCS through the real-time data communication link. The OCS monitors equipment operation status of data receiving stations and other subsystems in real time. Automatic or manual scheduling commands will be ordered according to operation plan, to control the business operation of each subsystem.

The man-machine interaction of OCS is use distributed processing and centralized monitoring mode based on $\mathrm{C} / \mathrm{S}+\mathrm{B} / \mathrm{S}$ frame. All users requirements are send from the computer terminal, which installed the centralized monitoring client software. Assign service to the host according to predefined policies and plans. Establish logical links between the server and client. The server executes the program according to requirement, and feedback the processing results to client through the logical link.

\section{Conclusion}

The design scheme has been implemented in the satellite ground application system. As the nerve center of the ground system, it is responsible for the task operation scheduling and the state monitoring. OCS system can used in the complexity object and multi-level tasks based on the hierarchical scheduling model. The scheme design of application system based on UNIX is conducive to control the satellite at ground.

\section{References}

[1] Bruce Lee, MAO Wenlin. Software architecture and design of the pipe-filter mode [J]. Computer engineering and applications, 2003,39 (35) : 114-115.

[2] Bing Zhou, Shi-Mei Su. A Rate Control Scheme of the Even Low Bit-Rate Video Encoder [J]. International Journal of Distributed Sensor Networks, 2009, 5(1).

[3] Caloto Teresa. Quality control and data-handling in multicentre studies: the case of the Multicentre Project for Tuberculosis Research [J]. BMC Medical Research Methodology, 2001, $1(1)$.

[4] Zou Yong, Li Mingshu, Wang Qing. Scheduling theory and method of open real-time[J]. Journal of software, 2003,14 (1) : 83-90.

[5] Yun TIAN, Shiqi GAO. Transonic buffet control research with two types of shock control bump based on RAE2822 airfoil[J]. Chinese Journal of Aeronautics, 2017. 